\title{
Computational Model to Probe Cellular Mechanics during Epithelial-Mesenchymal Transition
}

\author{
Diego A. Vargas Oliver Bates Muhammad H. Zaman \\ Department of Biomedical Engineering, Boston University, Boston, Mass., USA
}

\section{Key Words}

Epithelial to mesenchymal transition · Cadherin switch · Cell migration · Computational modeling · Agent-based model . Epithelium disruption

\begin{abstract}
During the epithelial to mesenchymal transition (EMT), polarized cells in the epithelium can undergo a transformation characterized by the loss of cell-cell junctions and increased migratory activity into nonpolarized invasive cells. These cells adopt a mesenchymal shape and migrate into the basal lamina. Such transitions have been observed in developmental processes and have been linked to cancer cell metastasis. Most experimental studies on EMT search for molecular markers indicating an epithelial or mesenchymal conformation, focussing on afferent signaling pathways received by cells undergoing this transformation; however, these approaches are unable to track mechanical changes in the cell and the possible role this plays in EMT. In order to address this gap in our understanding, we have used a quantitative approach to study population level effects of single cell changes typically occurring during EMT. We have developed a computational model making use of the advantages of both single cell migratory models and agent-based cell population models to study the effect of cellular molecular processes in EMT. The disruption of a cell sheet representing the epithelium over a dense extracellular matrix (ECM) is simulated using interaction forces between different cells and between cells and discrete fibers representing the ECM. In our
\end{abstract}

study, two different parameters were varied: protrusion force magnitude and E-cadherin (cell-cell junction) concentration. The cell population was tracked for 3 days and the number of cells that leave the layer, the depth of invasion,

\section{Abbreviations used in this paper}

CC constant cadherin concentration

DC dynamic cadherin concentration

ECM extracellular matrix

EMT epithelial to mesenchymal transition

HF high force

LF low force

D.A.V. and O.B. contributed equally to this work.

This paper is from the 5th International EMT Meeting, Singapore, October 10-13, 2011.

Further articles in this special topic series: 1. Newgreen, D.F., E.W. Thompson (2013) Progress in epithelial-mesenchymal transition research. Cells Tissues Organs 197: 421-423. 2. Said, N.A.B.M., K.J. Simpson, E.D. Williams (2013) Strategies and challenges for systematically mapping biologically significant molecular pathways regulating carcinoma epithelial-mesenchymal transition. Cells Tissues Organs 197: 424-434. 3. Elsum, I.A., P.O. Humbert (2013) Localization, not important in all tumor-suppressing properties: a lesson learnt from Scribble. Cells Tissues Organs 198: 1-11. 4. Kulesa, P.M., J.A. Morrison, C.M. Bailey (2013) The neural crest and cancer: a developmental spin on melanoma. Cells Tissues Organs 198: 12-21.

\section{KARGER}

E-Mail karger@karger.com

www.karger.com/cto
(C) 2013 S. Karger AG, Basel

$1422-6405 / 13 / 1976-0435 \$ 38.00 / 0$
Dr. Muhammad H. Zaman

Department of Biomedical Engineering, Boston University

44 Cummington Mall

Boston, MA 02215 (USA)

E-Mail zaman@bu.edu 
and the percentage of initial number of cells that remain in the layer (a measure of epithelium disruption) were monitored. Our studies suggest that having a high protrusion force or a reduction in cell-cell attachments is enough to cause EMT. Our results also demonstrate that the morphological progression in membrane disruption has an effect on the number of cells becoming invasive, with epithelial layers broken into clusters hindering the further exodus of cells. The results reveal the quantitative interplay between two key parameters involved in EMT and suggest potential avenues for further exploration of a systems level understanding of EMT.

Copyright $\odot 2013$ S. Karger AG, Basel

\section{Introduction}

During development of an organism or repair of many tissues, the ability of cells to change their behavior is crucial; cells can differentiate and dedifferentiate based on environmental cues to fulfill different tasks at different times. Although both differentiation and migration are well known and studied processes, the exact quantitative nature of their relationship remains elusive. One example of this relationship is seen in the epithelial to mesenchymal transition (EMT). In EMT, polarized cells in the epithelium can undergo a transformation characterized by the loss of cell-cell junctions and increased migratory activity into nonpolarized invasive cells [Yilmaz and Christofori, 2009]. EMT was initially recognized during embryonic development, when it was treated as synonymous with the process of delamination, where the neural crest separates from its neighboring neuroepithelial cells [Theveneau and Mayor, 2012]. While EMT appears to be a crucial part of delamination, this transition is also seen in other cellular processes of high physiological significance. For example, EMT has also been associated with the process of cancer metastasis, where cancerous cells leave the primary lesion and migrate toward nearby blood or lymphatic vessels to intravasate and invade distant sites in the organism [Geiger et al., 2008].

In contrast to embryonic development, where cellular response appears to be orchestrated, cancer metastasis in general is not characterized by sequential events but instead by a multistep clonal selection [Chaffer and Weinberg, 2011]. The complexity of the changes that a cell must undergo to alter its phenotype reveals how difficult it is to define EMT specifically. These changes affect mechanical properties of the cytoskeleton, cell-environment and cell-cell signaling, as well as genetic regulation. Fur- thermore, these are highly interrelated aspects of cellular life, governed by complicated quantitative relationships. It is for this reason that we believe in silico experiments may provide an avenue of investigation into EMT. Computer simulations provide the ability to look at a breadth of processes that may be inaccessible experimentally. Additionally, these simulations can also explain qualitative phenomena or provide guidance for future experiments. Finally, they provide a platform to study in vivo complexity.

\section{Intracellular Molecular Mechanisms of EMT}

One of the more characteristic changes cells undergo during EMT is the reduction of cell-cell adhesions [Hirohashi and Kanai, 2003; Geiger et al., 2008]. Cells in the epithelium typically express the adhesion molecule Ecadherin, whereas mesenchymal cells express $\mathrm{N}$-cadherin. This change is known as the cadherin switch. It can be induced by either intrinsic or extrinsic signals [Yilmaz and Christofori, 2009]. This step is necessary for epithelial coherence to be lost. In addition to making cells less adhesive and thus capable of detachment from the epithelial layer, it also affects the internal properties of the cells themselves.

Although the list of molecules involved in this connection is still under review, it is believed that E-cadherin is connected to intracellular actin filaments (F-actin) by multiple pathways. More specifically, it is believed that E-cadherin is associated with $\beta$-catenin. This complex may then interact with F-actin either through $\alpha$-catenin or eplin. Alternatively, the complex can interact with p120-catenin, which represses the activity of RhoA and activates Rac1; both actions aid cell migration by contributing to the formation of migratory membrane protrusions [Wildenberg et al., 2006]. In general, downregulation of E-cadherin has been found to coincide with the accumulation of $\beta$-catenin in the nucleus, where it is believed to interact with transcription factors causing the cell to migrate by modulating the expression of molecules such as L1-CAM, matrix-metalloproteases and fascin [Ramis-Conde et al., 2008].

In addition to disrupting E-cadherin junctions, there is also a reduction in desmosomes during EMT. This reduction correlates with a change in the composition of the cytoskeleton: the intermediate filaments go from containing the protein cytokeratin to having vimentin [Geiger et al., 2008]. Little information is available on the consequences of this cytoskeletal change in the context of EMT. Yet vimentin has been found to be involved in signal transduction between cells and cause decreased cel- 
lular contractibility [Wang and Stamenovic, 2002] and contribute towards cellular mechanical stability, motility and chemotaxis [Eckes et al., 1998; Mendez et al., 2010], all of which would potentially affect the integrity of an epithelial layer.

\section{Computer Modeling of Cell Populations}

The biophysical and molecular processes involved in single cell migration have been studied for decades and are relatively well understood [Lauffenburger and Horwitz, 1996]. In recent years, numerous models for single cell migration have emerged and shown success in reproducing cell morphology, directionality, persistence and velocity, as a function of cellular and environmental properties [Zaman et al., 2005; Harjanto and Zaman, 2010]. Contrary to single cell motion, our understanding of collective cell motion is largely qualitative. It has been observed in both in vivo models [Parker et al., 2004; Ghysen and Dambly-Chaudiere, 2007; Blum et al., 2008] and in vitro assays [Rørth, 2009], yet modeling efforts to date have failed to elucidate the mechanisms underlying this phenomenon. Part of the difficulty in understanding collective cell migration is that it is inherently a multi-scale phenomenon. There are processes occurring on many levels ranging from the molecular level (within a specific cell) to population level, where overall organization and pattern formation is observed. For this reason, most models tend to focus on elucidating the role of a single factor, for instance cell-cell adhesion [Armstrong et al., 2006]. Many of these models have been developed with cancer in mind. Gerisch and Chaplain [2008], for example, looked at the effect of cell adhesion on cancer invasion. In another paper, Painter [2009] looked at the role of the extracellular matrix (ECM) fiber orientation in cell migration. Finally, Jiao and Torquato [2011] examined the role of ECM degradation in cancer invasion and tumor growth.

While single and collective cell motion has been modeled to some extent, little has been done to study EMT from a computational perspective. Among the few studies reported, Ramis-Conde et al. [2008] briefly present an application of an agent-based model of tumor growth in the context of EMT; they model the kinetics of the E-cadherin$\beta$-catenin pathway and track waves of a rise in intracellular $\beta$-catenin concentration across cellular populations in a sheet. When briefly applying their model to a migratory population, the potential for disruption of the epithelial layer is shown, but they abstain from quantifying their results in this regard.

Cellular Mechanics in EMT
Cell population models to date can be classified into two main categories: agent-based models and continuum models. Agent-based models are those which treat cells as individual units interacting according to a set of rules, often including a stochastic element [Drasdo and Hohme, 2005]. Continuum models on the other hand are deterministic in nature, often defined by a set of analytic equations. These treat cell populations as a whole, and have the ability to represent larger systems, yet lack the ability to effectively take into account changing intercellular interactions [Drasdo and Hohme, 2005]. For instance, cell clusters modeled as a continuum lack the ability to breakup or combine into new groups. In contrast, when modeling tumor growth, there are many examples where cells are represented by individually interacting objects [Anderson, 2005; Schaller and Meyer-Hermann, 2005; Drasdo, 2007]; however, in these instances, the main interest is in the tumor itself, therefore cell migration and detachment is not considered outside of the primary mass of cells.

With the present work, we develop a new model to take advantage of both single cell migratory models and agentbased cell population models to show the potential of in silico experiments in the study of EMT and gain mechanistic and mechanical insights into the behavior of cellular clusters undergoing EMT. Special emphasis is put on presenting the model parameters that can be related to the molecular processes that coordinate and affect cellular behavior and interactions during EMT.

\section{Methods}

\section{Model Formulation}

Similar to some of the agent-based models by Schaller and Meyer-Hermann [2005] and Drasdo [2007], our model keeps track of cells individually using a Delaunay triangulation to partition space and also keeps track of the neighboring relations among cells. We model each cell as a sphere with an elastic spherical core with a radius of $75 \%$ of the total cell radius. This allows cells to come into contact with the cellular areas of interaction approximated based on spherical contacts while preventing cells from occupying the same space due to the repulsion forces that come into action when the elastic cores come together.

In addition to the position of the center of the elastic sphere, each cell is defined by its radius and concentration of cell membrane receptors $\left(c_{r e c}\right)$ and integrin concentration $\left(c_{i n t}\right) . c_{r e c}$ and $c_{i n t}$ are normalized relative to a maximum concentration, and therefore vary from 0 to 1 . This maximum concentration is encapsulated by the adhesion force coefficient, $c_{r e c}$ represents the relative presence of generic receptors and ligands for cell-cell interaction (i.e. $c_{r e c}$ can be taken to be the E-cadherin concentration in the simulations here presented), while $c_{i n t}$ represents the relative concentration of generic integrins that interact with the ECM for cel- 
lular migration. Cell proliferation is ignored in these simulations to isolate the effects of single cell properties.

Our model also includes the ECM into the framework as it plays an important part in cell migration influencing signaling within the cell and as the substrate over which cells exert forces for motion. The ECM is modeled as a set of discrete fibers, making our approach novel and unique as compared to other studies where ECM is modeled as a continuum. We initialize the fiber network by creating a cubic lattice where all points are connected by cylinders; the positions of the lattice points are shifted in a random direction by the same distance, and then each fiber is potentially cut based on probability. The radius of each fiber is kept constant because the area of interaction between cells and fibers is important in determining cellular traction forces; however, it can potentially be varied to simulate a more heterogeneous ECM. Each fiber has ligand concentration ( $c_{\text {lig }}$; normalized relative to a maximal value) associated with it, to which cells can attach. This quantity was also kept constant. Fibers do not move or change throughout the simulations.

A force-based dynamics approach, similar to previously developed models [Zaman et al., 2005; Harjanto and Zaman, 2010; Vargas and Zaman, 2011], was used to define motion of the cells. Each time step simulates the motion of each cell over $30 \mathrm{~s}$, during which all forces acting on the cell are summed. The sum of the forces is described by equation 1 :

$$
F_{t o t}=F_{p r o t}+F_{e l}+F_{f i b}+F_{d e t}
$$

$F_{\text {prot }}$ accounts for the small protrusion forces due to the randomly directed ruffling of the cell membrane and the traction force generated by each cell due to its attachments to the ECM fibers. Its initial direction is chosen at random at the beginning of each step and its total magnitude is constant; the magnitude was changed in different simulations (discussed later). Because this force would only lead to movement if there is a substrate for the cell to exert force on, it is broken down into components by projecting the force onto the planes of each ECM fiber in contact with the cell $\left(F_{\text {pproj }}\right)$. At each point where force is exerted, a traction force opposing it is generated, so the projections are scaled based on two factors affecting traction. The first factor $\left(A_{r}\right)$ is the ratio of the cell surface area of interaction with a particular attachment point (with a particular fiber) over the area of interaction with all fibers. The second factor $(\beta)$ is an adhesivity parameter similar to that used by Zaman et al. [2005] defined as the product of ligand concentration on the fiber and integrin concentration on the cell. Once scaled, the resulting forces are added to obtain the final protrusion force acting on the cell. Equations 2 and 3 express the calculation of this force for a cell; the subscript $f$ is used to describe the quantity for a specific fiber in contact with the cell:

$$
\begin{aligned}
& \boldsymbol{F}_{\text {prot }}=\sum_{f=1}^{N} \beta_{f} A_{r, f} \boldsymbol{F}_{\text {pproj } f} \\
& \beta_{f}=c_{\text {int }} c_{\text {lig }, f}
\end{aligned}
$$

$F_{e l}$ represents the elastic repulsion forces that come into action if two cells come too close together. Equation 4, from contact mechanics, has been used in cell models [Schaller and Meyer-Hermann, 2005] and describes the magnitude of the elastic repulsion force for two spheres $i$ and $j$ (in this case two cells):

$$
\left|F_{e l}\right|=\frac{h_{i j}^{3 / 2}}{\frac{3}{4}\left(\frac{1-v_{i}^{2}}{E_{i}}+\frac{1-v_{j}^{2}}{E_{j}}\right) \sqrt{\frac{1}{R_{i}}+\frac{1}{R_{j}}}}
$$

where $h_{i j}$ represents the maximum overlap sphere $i$ and $j$ would have if they penetrate each other instead of deforming, $E$ is the Young's modulus of the elastic mini-sphere, $v$ is the Poisson ratio and $R$ is the radius. Similarly, $F_{f i b}$ represents the elastic repulsion force that comes into action if a cell and a fiber come too close together. Unlike cells, fibers are considered to be all rigid (no elastic core) and have a higher Young's modulus. This force prevents cells from moving across fibers. Its magnitude was approximated by calculating it with equation 4 without using an elastic core for the fiber but using the actual radius of the fiber for $R$.

Finally, $\boldsymbol{F}_{d e t}$ is a small force acting on each cell pushing it towards all fibers with which it is in contact. Its magnitude is $10 \%$ of the projection of $\boldsymbol{F}_{\text {prot }}$ in the cell-fiber direction (closest distance); it is also scaled based on $A_{r}$ and $\beta$. This force, with its small magnitude, is meant to complement the pulling force moving each cell along a fiber since a cell cannot move along a fiber without attaching to it and changing shape. This force also prevented cells from becoming detached from the ECM in cases where elastic repulsion was high.

The displacement of each cell is calculated based on the total force acting on the cell. By taking into account that the Reynolds numbers for cellular motion in tissue are very small, an overdamped approximation is used, and the total force felt by the cell is related to the displacement by equation 5 , where $r$ is cell position [Schaller and Meyer-Hermann, 2005; Zaman et al., 2005; Harjanto et al., 2011].

$$
\dot{\boldsymbol{r}}=\frac{\boldsymbol{F}_{\text {tot }}}{\gamma_{\text {tot }}}
$$

$\gamma_{\text {tot }}$ represents the friction experienced by the cells upon motion. This term is a sum of multiple friction terms because hindrance in motion can be attributed to cell-cell attachments $\left(\gamma_{\text {cell }}\right)$, cell-fiber attachments $\left(\gamma_{f i b}\right)$ and viscosity of the environment $\left(\gamma_{v i s c}\right)$ due to extracellular fluid, hyaluronic acid, other glycosaminoglycans and smaller fibrillar components of the ECM. These are defined in equations 6,7 and 8 for a cell $i$, where $j$ and $f$ are subindices to indicate adjacent cells and touching fibers, respectively:

$$
\begin{aligned}
& \gamma_{\text {cell } i}=\gamma_{\max } \sum_{j=1}^{N} A_{s, i j} \frac{1}{2}\left(1-\frac{\boldsymbol{F}_{\text {tot } i} \cdot \boldsymbol{n}_{i j}}{\left|\boldsymbol{F}_{\text {tot } i}\right|}\right)\left(c_{r e c, i} c_{r e c, j}\right) \\
& \gamma_{f i b i}=\sum_{f=1}^{N} \varphi A_{r, f} c_{i n t, i} c_{\text {lig }, f} \\
& \gamma_{v i s c}=6 \pi \eta r_{0}
\end{aligned}
$$

Equation 6 was taken from the work by Schaller and Meyer-Hermann [2005]; here $A_{s, i j}$ is the contact surface area of the two spheres $i$ and $j, \gamma_{\max }$ is a friction parameter and $n_{i j}$ is the vector from the center of cell $i$ to cell $j$. In equation 7, $\varphi$ is a parameter representing the contribution to friction by each ligand-integrin bond. Following the Stokes-Einstein equation, because cells are modeled partly by spheres, equation 8 uses the viscous resistance of the ECM, $\eta$. The values as well as the units for these and all other parameters used in the model are displayed in table 1. 
Fig. 1. Initial configuration of cells, shown in red, in a hexagonal lattice forming a sheet (left) over discrete fibers, shown in cyan, representing the ECM (right).

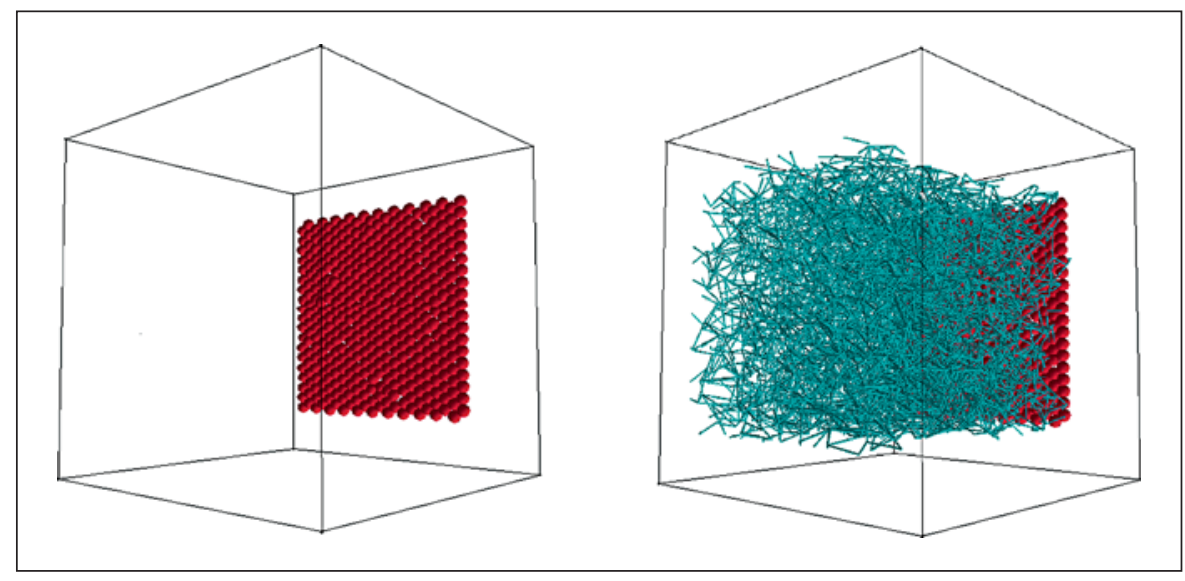

Table 1. Parameter values for force calculations in the model

\begin{tabular}{llll}
\hline Parameter & Value & Units & Reference \\
\hline Integrin concentration on cell, $c_{\text {int }}$ & 1.0 & $\mathrm{No.}$ & Fixed \\
Ligand concentration on fiber, $c_{l i g}$ & 0.5 & $\mathrm{No}$ & Fixed \\
Cellular (mini sphere) Young's modulus, $E$ & $5 \times 10^{-3}$ & $\mathrm{MPa}$ & [Galle et al., 2005], estimate \\
Fibrillar Young's modulus, $E$ & $1 \times 10^{-2}$ & $\mathrm{MPa}$ & Assumption \\
Cellular (mini sphere) Poisson ratio, $v$ & 0.5 & $\mathrm{No}$ & Assumption \\
Fibrillar Poisson ratio, $v$ & 0.5 & $\mathrm{No}$ & Assumption \\
Cell-cell friction parameter, $\gamma_{\max }$ & $5 \times 10^{-3}$ & $\mathrm{~kg} / \mu \mathrm{m}^{2} / \mathrm{s}$ & Fit \\
Ligand-integrin friction per bond, $\varphi$ & 1.0 & $\mathrm{~kg} / \mathrm{s}$ & [Zaman et al., 2005], estimate \\
Viscosity of ECM, $\eta$ & $1 \times 10^{-3}$ & $\mathrm{~kg} / \mu \mathrm{m} / \mathrm{s}$ & [Galle et al., 2005], estimate \\
\hline
\end{tabular}

Before each cell is moved, there is a check in place to see if the cell would potentially be able to move from the new position (i.e. whether a fiber is close enough for the cell to reach it with its protrusions). If no fiber is within the cell radius, then the movement is recalculated without the protrusion force, only the elastic forces with respect to other cells and fibers are taken into account, and the actual new position is calculated. This part of the simulation makes the assumption that a cell moves no more than as far as it could prod with its membrane projections; this is particularly true when the time step chosen $(30 \mathrm{~s})$ is equivalent to a fraction of the cell's total migration cycle ( $600 \mathrm{~s})$.

\section{Model Setup and Simulations}

The system is initialized by creating a sheet of cells representing the epithelial layer over a dense set of fibers representing the basal lamina and ECM underneath the epithelium. In the plane of the sheet (xy-plane), cells are placed in a hexagonal lattice equidistant from each other at first. The cells have a radius of $r_{0}=4.9 \mu \mathrm{m}$, and each cell is placed at $0.98 r_{0} \sqrt{ } 3 \mu \mathrm{m}$ from each other and then moved randomly in both the $\mathrm{x}$ - and $\mathrm{y}$-positions by a randomized fraction of $0.5 \mu \mathrm{m}$. Similarly, their $\mathrm{z}$-position is randomized within $1 \mu \mathrm{m}$ of the plane. It is the initial $\mathrm{z}$-position before randomization of the cells that is used to track invasion of the invasive cells leaving the layer. The layer is formed by 418 cells and is close to squared; cells at the edge of the sheet are not allowed to move. This initial configuration was chosen such that cells would all start tightly packed to prevent the sheet from losing its shape by expanding its boundaries.

The fiber network was also initialized at the beginning of every simulation. Figure 1 depicts this initial configuration. The lattice points defining the fiber network were positioned $11 \mu \mathrm{m}$ from each other and displaced by $9 \mu \mathrm{m}$. There was a $30 \%$ chance of each fiber being cut. These numbers were chosen such that the resulting fiber network would have an approximate average fiber length of $15 \mu \mathrm{m}$ as was found for collagen gels used in in vitro cell migration studies by confocal reflectance microscopy [Harjanto et al., 2011]. This resulted in approximately 8,000 fibers in a space of approximately $5 \times 10^{-3} \mathrm{~mm}^{3}$. The extent of the matrix in $\mathrm{x}$ - and $\mathrm{y}$-directions was the same as the epithelial layer, and it was $200 \mu \mathrm{m}$ in the $\mathrm{z}$-direction (arbitrary choice such that no cell would migrate to the end of the matrix in this direction). The radius of the fibers was chosen to be $0.5 \mu \mathrm{m}$.

Each simulation consisted in setting up the epithelial layer over the ECM and letting the cells move for 8,640 steps, which is equivalent to 3 days. Two different parameters were changed in the different simulations: protrusion force magnitude and E-cadherin concentration control. Two different constant protrusion forces $\left(\boldsymbol{F}_{\text {prot }}\right)$ were used, defined from now on as the low force (LF) and 


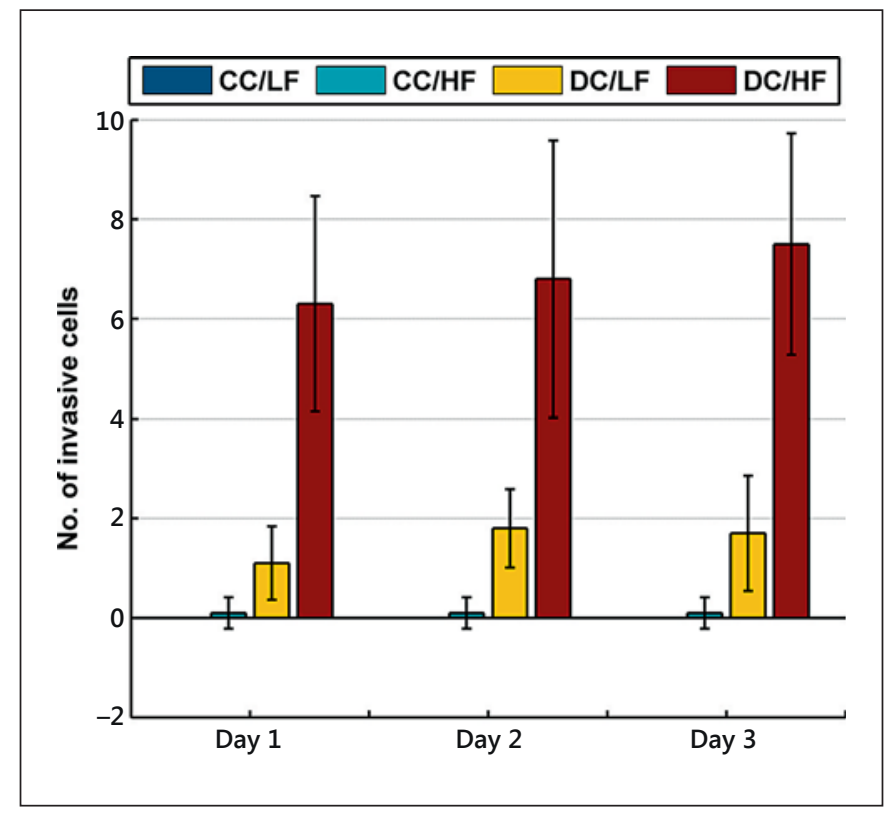

Fig. 2. Average number of cells that left the epithelial layer at 1,2 and 3 days for the four different conditions. The average was made over ten simulations. Error bars represent 1 standard deviation.

high force (HF): $F_{\text {low }}=0.0025 \mu \mathrm{N}$ and $F_{\text {high }}=0.005 \mu \mathrm{N}$. These values were chosen based on observations made when running simulations with a similar model but studying tumor growth [unpubl. data]: $F_{\text {low }}$ represents the lowest with which a cell may detach from a cell mass (e.g. tumor or cell sheet) and $F_{\text {high }}$ was found to be close to the highest possible force with which cells move without causing high repulsion elastic forces with fibers that may not accurately represent cellular motion. The E-cadherin concentration was either left constant at $c_{\text {rec }}=1.0$ or allowed to vary for each cell moved based on the changing total surface area of interaction with all neighboring cells as described by equation 9: these settings will be referred to from here on as constant cadherin concentration (CC) and dynamic cadherin concentration (DC), respectively.

$$
\partial c_{r e c}=\sqrt{\frac{d A_{s}}{4 \pi r_{0}^{2}}}
$$

where $d A_{s}$ is the change in area of interaction with all neighboring cells at each step. $4 \pi r_{0}^{2}$ represents the maximum change in the area of interaction, the surface area of the cell. By performing all combinations of the force magnitude and the cadherin concentrations, four different sets of simulations were run. Each simulation was run ten times and three quantities were measured every 2,880 steps (1 day): (1) the number of cells that leave the layer (defined as the number of detached cells at least at three cell radii distance from the initial z-position); (2) the percentage of the initial number of cells that remain in the layer (defined as the number of cells within $2 \mu \mathrm{m}$ from the initial layer z-position over the initial number of cells in the layer) - this is meant as an approximate measure of epithelial layer disruption; (3) average depth of penetration of invasive cells.

\section{Results}

First, we observed the number of invasive cells that detached from the cell sheet. Based on the selected values of $F_{\text {low }}$ and $F_{\text {high }}$, little detachment from the sheet was expected for the constant cadherin and $F_{\text {low }}(\mathrm{CC} / \mathrm{LF})$ and dynamic cadherin and $F_{\text {low }}$ (DC/LF) conditions, while some detachment was expected for the constant cadherin and $F_{\text {high }}(\mathrm{CC} / \mathrm{HF})$ and dynamic cadherin and $F_{\text {high }}(\mathrm{DC} /$ $\mathrm{HF}$ ) conditions. Figure 2 shows the number of cells that detached from the epithelial layer and were able to make it a distance of at least three cell radii from the initial position in the z-direction (into the fiber network).

Our results show how having a high protrusion force or being able to reduce, even temporarily, cell-cell attachments is enough to cause a cell to leave the epithelial layer. Figure 2 shows how individually these characteristics could be enough to trigger cellular exodus. We note that no cell left the layer in the CC/LF condition. We also observed that in all simulations with the $\mathrm{CC} / \mathrm{HF}$ condition, only a single cell left the epithelial sheet in a single simulation. This suggests that a cell losing its cadherin junctions is more relevant to EMT than increased migration activity; however, the average number of invasive cells, taking into account the error bars, is very close in the CC/ $\mathrm{HF}$ and DC/LF conditions.

Our simulations also suggest that there is no significant increase in the number of invasive cells as a function of time; most cells leave the epithelial sheet in the first day. The progression of epithelial sheet deformation reveals a possible reason for this observation. Figure 3 shows images of the system at day 3 for each of the four conditions used in the simulations. We note that the layer seems to break up into clusters of cells as a function of time. The progression of cell detachment with time may suggest that a cell is more likely to detach from a single-cell layer than a circular cluster. This can be explained by the difference in total contact area between cells in a sheet versus in a circular cluster. Further investigation into the total contact area as well as the total force acting on cell versus time could elucidate the mechanism. One important point to keep in mind is that this may be a model-specific observation that does not hold true if cells are not modeled as elastic spheres. This again is an interesting question to probe in the future. The progressive clustering of cells in the sheet seems to be more drastic in the simulations where cells move with the high protrusion force $\left(F_{\text {high }}\right)$ and especially with the CC/HF condition (fig. $3 \mathrm{~b}$ ). When the protrusion force is high but cells still have strong cell-cell junctions, it may be more likely for cells to 
Fig. 3. Example of final conformation of the cell sheet at day 3 for the four different conditions used in the simulations: $\mathrm{CC} / \mathrm{LF}$ (a), CC/HF (b), DC/LF (c) and DC/HF (d). Red cells are under tension above a certain value $(1 \mathrm{~Pa})$ and less likely to become loose than green cells, with tension below this value.

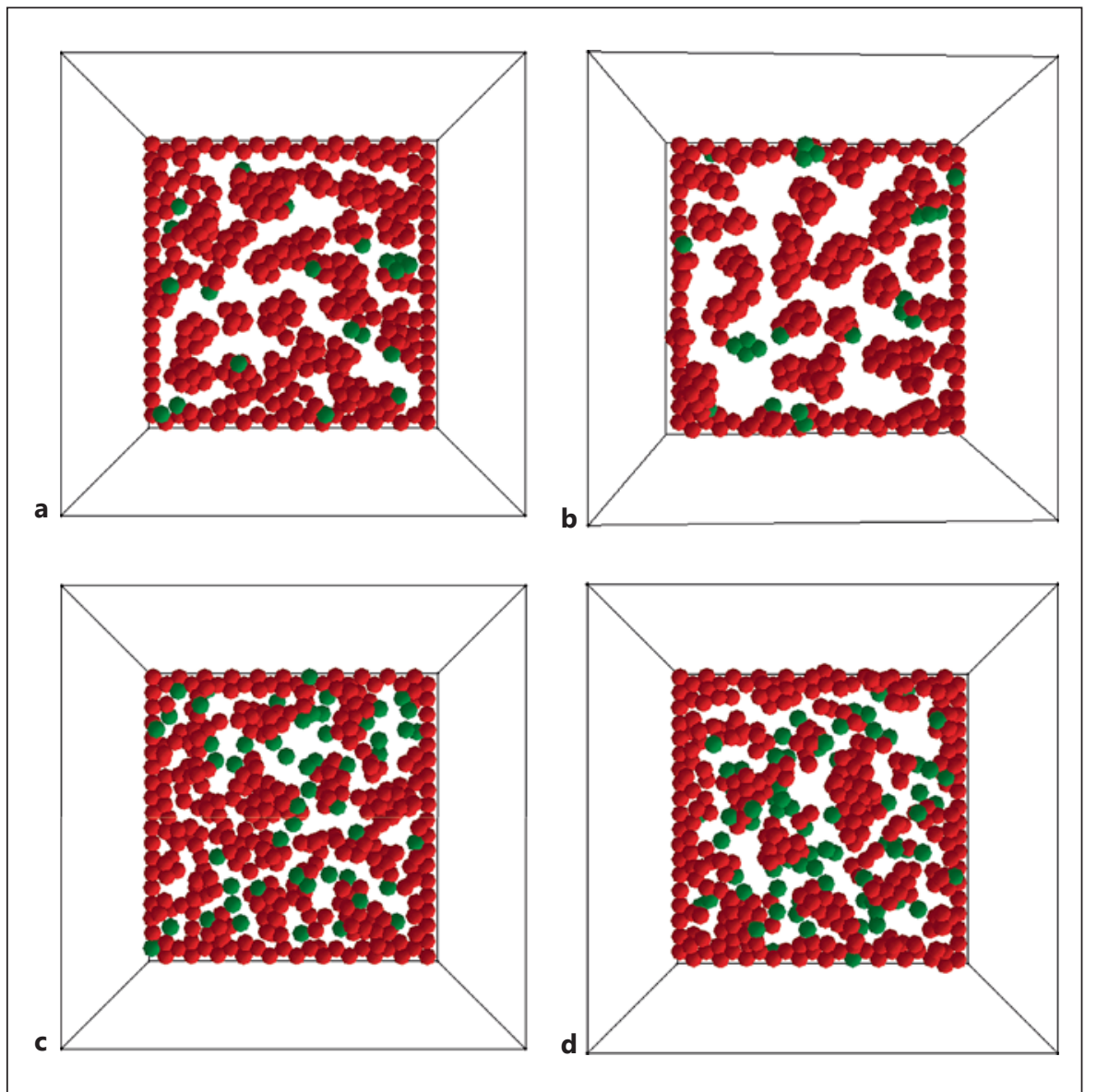

move against other cells than away into the ECM. More generally, figure 4 shows the average membrane disruption for each condition and characterizes this shape change for all simulations. Taking into account the observed clustering, it is not immediately obvious that at every checkpoint (each day) the average percentages are practically equal for simulations where cells have the same protrusion force independently of cadherin concentration. This shows it is the high protrusion force that causes the clusters to grow in the $\mathrm{z}$-direction away from the ECM and pushes cells off the plane even when a high and constant cadherin expression leads to clustering.

It is important to note that membrane disruption due to single cell migration into the ECM appears to be minimal. We noticed that even in cases where a relatively high number of cells leave the sheet, it is merely 2\% of all cells; most of the disruption is due to cell rearrangement within the sheet. While figure 2 shows no significant change at each checkpoint after day 1 , figure 4 shows a steady decline for all conditions in the average percentage of cells remaining in the layer. This indicates that

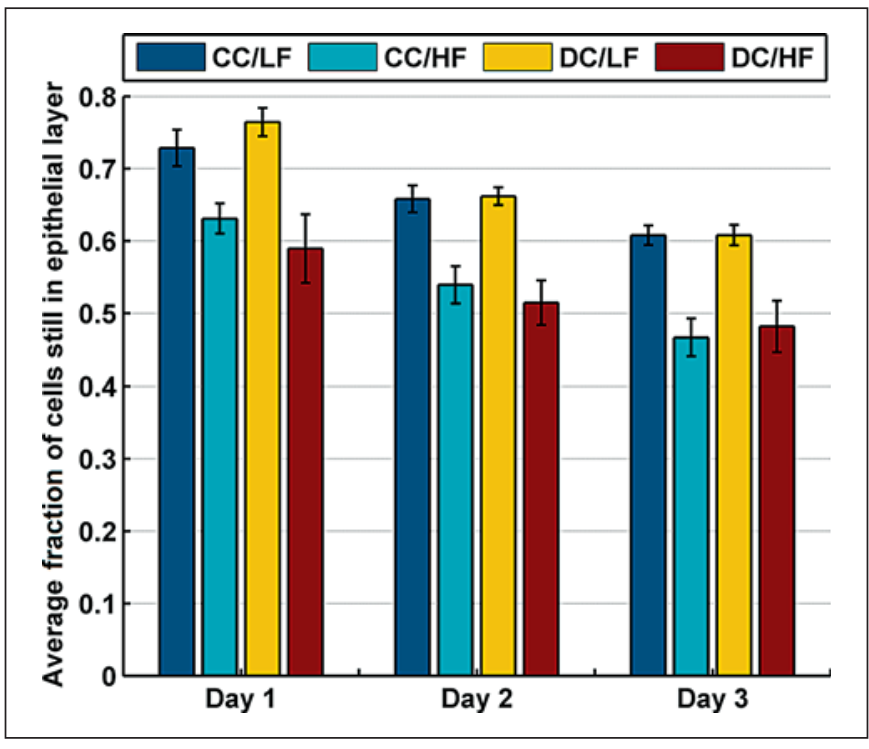

Fig. 4. Average percentage of cells within $2 \mu \mathrm{m}$ from initial layer $\mathrm{z}$-position at 1,2 and 3 days for the four different conditions. The average was made over ten simulations. Error bars represent 1 standard deviation. 


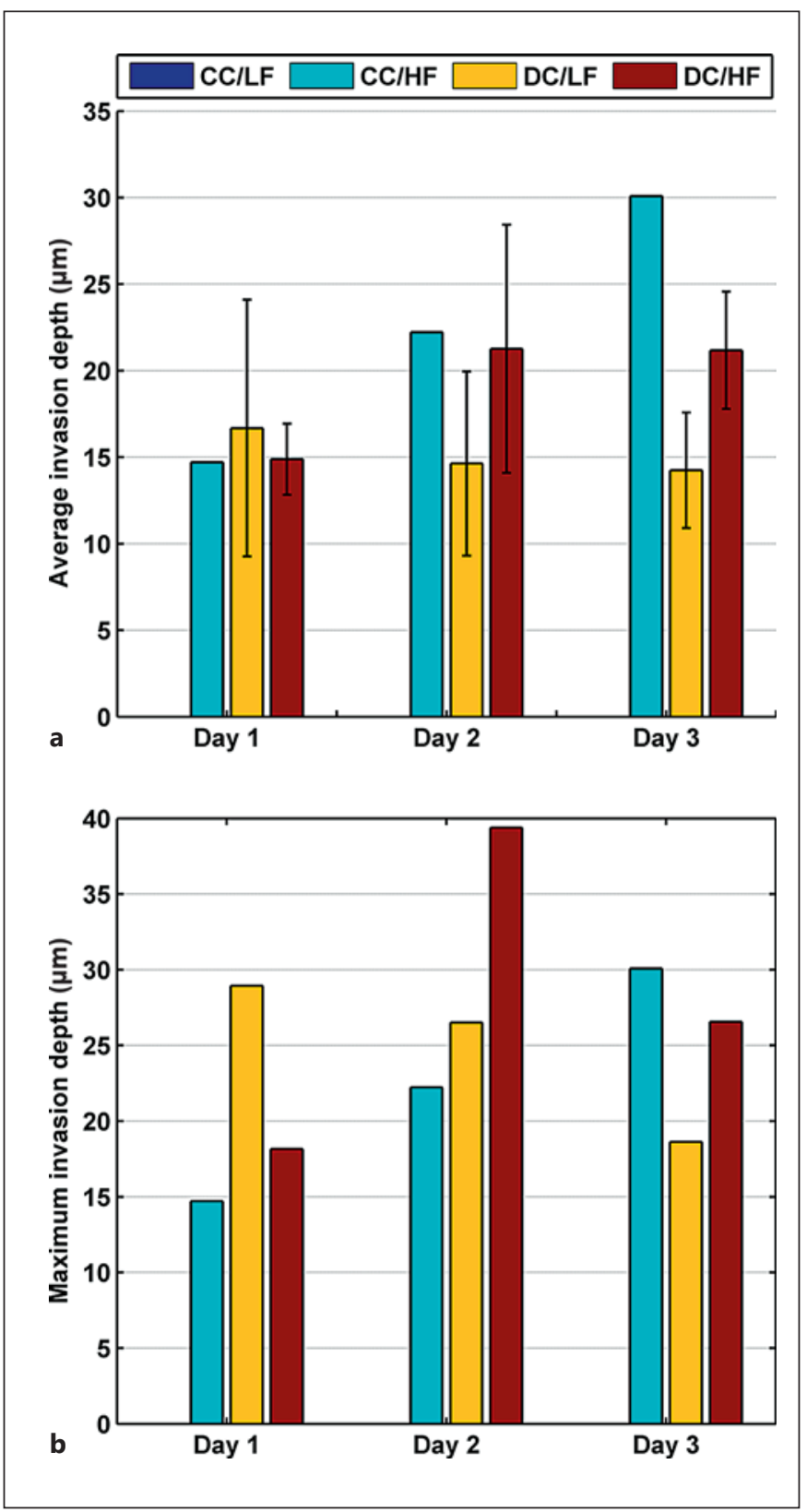

Fig. 5. Average invasion depth into fiber network of cells that left the epithelial layer at 1,2 and 3 days for the four different conditions (a). The average was made over ten simulations (error bars represent 1 standard deviation), and maximum invasion depth for all simulations at 1,2 and 3 days for the four different conditions (b).

while cells are active throughout the simulation they can only escape the sheet in the first part of it. It is much harder for a cell to leave a cluster than a sheet because the part of its surface area that interacts with other cells is much higher than when in a sheet. This new position within the cell collective hinders its motion due to the increased cell-cell attachments as well as the more limited access to fibers. Nonetheless, even if cells cannot move as easily individually, those at the edges of a cluster do impart force and, due to volume exclusion, the repulsion forces continue to push the cluster away from the fiber network causing the progressive drop of the quantity tracked in figure 4 .

Next, we observed the average depth of migration into the fiber network by cells that leave the cell sheet. Figure 5 shows this along with the maximal penetration observed during all simulations for the four conditions mentioned. Most surprising was that the average invasion depth for simulations with the DC/LF condition was comparable to the conditions where cells attempted to move with the high protrusion force $\left(F_{\text {high }}\right)$. This can be attributed to the phenomenon of hindered transport, previously observed in studies of motion of spherical particles in fibrous media [Phillips et al., 1989]; this phenomenon consists of a deeper penetration by the particles in question when there are fewer other particles in its surroundings to collide hindering each other's movements. Also important is the fact that there is less layer disruption in the simulations where cells attempted to move with the low protrusion force $\left(F_{\text {low }}\right)$; it would be less likely for cells to return to the layer if the cell sheet itself has not clustered reaching deeper into the fiber network.

For simulations run with the CC/HF condition, we point out that even if it was only a single cell that left the cell sheet, by day 3 of the simulation this cell had strayed farther than any other cell in all simulations. This may be a factor of the lack of cells in the fiber network to slow it down. The values of this cell seem to fit within those obtained for the DC/HF condition. Both CC/HF and DC/ $\mathrm{HF}$ had the same level of layer disruption; this indicates that as long as the layer does not hinder motion and cells can get away, the protrusion force has a bigger effect than cell-cell attachments in determining migration. It shows that with a high protrusion force cells are displaced more in each step.

\section{Discussion and Conclusion}

In this study, we report the construction and implementation of an integrated computational model to study the effect of cellular and ECM mechanics on EMT. Overall, our results demonstrate that our model is capable of studying how several different factors defining cellular 
motion can affect (individually or cooperatively) the disruption of a cell layer over a dense fiber network. Our results also suggest that either mutating towards a more migratory phenotype or undergoing the cadherin switch is enough for cells to leave the epithelial layer, but that both occurring together act to amplify the total effect. While it appears that the increase in migratory phenotype has a much larger impact on overall layer disruption, this in fact can only occur in a significant way if cell-cell junctions are lost. This could suggest that in EMT, the cadherin switch is a more fundamental step in the process than an acquired aggressive migratory phenotype.

More importantly, we demonstrate the ability to model and test the relationship between these two parameters, which at the moment remain difficult to probe experimentally. Our model may further be used to experiment with different forms of interrelation (similar to equation 9), and may easily be extended to experiment with different parameters representing other physical or chemical properties of the cell or ECM (such as those listed in table 1). Such a model also allows for exploration of behavior of the system as a whole, not just on an individual cell basis. The results displayed in figure 4 raise the question as to whether or not EMT causes an epithelial layer disruption, since most of the disruption is due to cell rearrangement within the sheet. Other questions that go beyond single cell behavior include: how does paracrine signaling affect EMT, is there hindering of migration due to nutrient depletion, and how does a chemotactic agent change migration out of the epithelial layer? Although not presented here, the model has the potential for using a grid in space as a continuum to track the diffusion of substances in the intercellular space because diffusion of small particles is much faster than cellular motion. The concentrations of substances at any position can be interpolated from the concentration at the grid points. This is done in many continuum or hybrid models [Anderson, 2005; Schaller and Meyer-Hermann, 2005]. In this way the effect of environmental cues and external signals can be incorporated into the model. Concentrations of extrinsic signals that trigger EMT, such as transforming growth factor- $\beta$, hepatocyte growth factor, members of epidermal growth factor family, and fibroblast growth factor could be modeled, as well as their effect on individual cells, to explore how an epithelial cell population would react.

As stated above no cell proliferation was considered in the present simulations. In part this keeps the results generally applicable since increased proliferation is not seen in all instances of EMT. During development, cell prolif- eration is carefully regulated, while in cancer, EMT is thought to occur at neoplasms where there is increased proliferation [Tarin et al., 2005]. Nonetheless, the ability for cells to divide can be added easily to the model in future studies; this is already done in existent agent-based models [Drasdo and Hohme, 2005; Schaller and MeyerHermann, 2005].

The ECM is another important player in the study of EMT. The composition of the fibers and its effect on cellular pathways can be included within our model. It has been shown, for instance, in other models that ECM fiber orientation has an impact on overall cell migration [Painter, 2009]. It will be an interesting area of future work to expand upon this model to see how ECM orientation and organization affects EMT more specifically. Additionally, the integrin composition and concentration in each cell was kept constant, but an agent-based model presents great potential for research in this regard. In addition to the individual cell interaction with a random ECM, there is also the response this interaction has on the cell populations. Our results raise the question as to how much a mesenchymal cell is slowed down by other cells that have undergone EMT; studies have shown that nearby migrating endothelial cells can communicate through mechanical signals across a stiff ECM, many times driving the cells away from each other [Reinhart-King et al., 2008]. The novel notion of modeling a discrete ECM can be used to track the effect of cells on each other when coming in contact with the same fiber.

In general, EMT implies many more changes than those discussed here, including internal changes in the cell, the dynamics of which are more complicated than the model presented here. As these individual changes and the pathways involved are elucidated, a better understanding of the EMT phenomenon can be formed. In silico studies can provide a way to test the effects of each change in isolation or coupled with one another, similar to what was done here with cell-cell attachments and migration strength. We believe that these in silico studies will complement existing in vitro and in vivo models in developing a quantitative, rigorous and multi-scale understanding of complex EMT-related processes in cancer metastasis, neural crest delamination and other developmental processes.

\section{Acknowledgements}

We are grateful for the support of this work by the NIH grant R01CA132633 to M.H.Z. 


\section{References}

-Anderson, A. (2005) A hybrid mathematical model of solid tumour invasion: the importance of cell adhesion. Math Med Biol 22: 163-186.

-Armstrong, N.J., K.J. Painter, J.A. Sherratt (2006) A continuum approach to modelling cell-cell adhesion. J Theor Biol 243: 98-113.

Blum, Y., H. Belting, E. Ellerstdottir, L. Herwig, F. Lueders, M. Affolter (2008) Complex cell rearrangements during intersegmental vessel sprouting and vessel fusion in the zebrafish embryo. Dev Biol 316: 312-322.

-Chaffer, C., R. Weinberg (2011) A perspective on cancer cell metastasis. Science 331: 1559 1564.

Drasdo, D. (2007) Center-based single-cell models: an approach to multi-cellular organization based on a conceptual analogy to colloidal particles. Math Biosci Interac 3: 171-196.

Drasdo, D., S. Hohme (2005) A single-cell based model of tumor growth in vitro: monolayers and spheroids. Phys Biol 2: 133-147.

Eckes, B., D. Dogic, E. Colucci-Guyon, N. Wang, A. Maniotis, D. Ingber, A. Merckling, F. Langa, M. Aumailley, A. Delouvee, V. Koteliansky, C. Babinet, T. Krieg (1998) Impaired mechanical stability, migration and contractile capacity in vimentin-deficient fibroblasts. J Cell Sci 111: 1897-1907.

-Galle, J., M. Loeffler, D. Drasdo (2005) Modeling the effect of deregulated proliferation and apoptosis on the growth dynamics of epithelial cell populations in vitro. Biophys J 88: $62-$ 75.

Geiger, T., H. Sabanay, N. Kravchenko-Balasha, B. Geiger, A. Levitzki (2008) Anomalous features of EMT during keratinocyte transformation. PLOS One 3: 1-9.

Gerisch, A, M.A.J. Chaplain (2008) Mathematical modeling of cancer cell invasion of tissue: Lo$\mathrm{cal}$ and non-local models and the effect of adhesion. J Theo Biol 250: 684-704.
Ghysen, A., C. Dambly-Chaudiere (2007) The lateral line microcosmos. Genes Dev 21: 2118 2130 .

Harjanto, D., J. Maffei, M. Zaman (2011) Quantitative analysis of the effect of cancer invasiveness and collagen concentration on $3 \mathrm{D}$ matrix remodeling. PLOS One 6: 1-8.

Harjanto, D., M. Zaman (2010) Computational study of proteolysis-driven single cell migration in a three-dimensional matrix. Ann Biomed Eng 38: 1815-1825.

Hirohashi, S., Y. Kanai (2003) Cell adhesion system and human cancer morphogenesis. Cancer Sci 94: 575-581.

Jiao, Y., S. Torquato (2011) Emergent behaviors from a cellular automaton model for invasive tumor growth in heterogeneous microenvironments. PLoS Comput Biol 7: e1002314.

Lauffenburger, D., A. Horwitz (1996) Cell migration: a physically integrated molecular process. Cell 84: 359-369.

Mendez, M., S. Kojima, R. Goldman (2010) Vimentin induces changes in cell shape, motility, and adhesion during the epithelial to mesenchymal transition. FASEB J 24: 1838-1851.

Painter, K. (2009) Modelling cell migration strategies in the extracellular matrix. J Math Biol 58: 511-543.

Parker, L., M. Schmidt, S. Jin, A. Gray, D. Beis, T. Pham, G. Frantz, S. Palmieri, K. Hillan, D Stainier, F. de Sauvage, W. Ye (2004) The endothelial-cell-derived secreted factor Egfl7 regulates vascular tube formation. Nature 428: 754-758.

Phillips, R., W. Deen, J. Brady (1989) Hindered transport of spherical macromolecules in fibrous membranes and gels. AIChE J 35: 1761-1769.
Ramis-Conde, I., D. Drasdo, A. Anderson, M. Chaplain (2008) Modeling the influence of the E-cadherin- $\beta$-catenin pathway in cancer cell invasion: a multiscale approach. Biophys J 95: 155-165.

Reinhart-King, C., M. Dembo, D. Hammer (2008) Cell-cell mechanical communication through compliant substrates. Biophys J 95: 60446051.

Rørth, P. (2009) Collective cell migration. Annu Rev Cell Dev Biol 25: 407-429.

- Schaller, G., M. Meyer-Hermann (2005) Multicellular tumor spheroid in an off-lattice $\mathrm{Vo}$ ronoi-Delaunay cell model. Phys Rev E 71: $1-16$.

Tarin, D., E. Thompson, D. Newgreen (2005) The fallacy of epithelial mesenchymal transition in neoplasia. Cancer Res 65: 5996-6000.

Theveneau, E., R. Mayor (2012) Neural crest delamination: from epithelium-to-mesenchyme transition to collective cell migration. Dev Biol 366: 34-54.

Vargas, D., M. Zaman (2011) Computational model for migration of a cell cluster in threedimensional matrices. Ann Biomed Eng 39: 2068-2079.

Wang, N., D. Stamenovic (2002) Mechanics of vimentin intermediate filaments. J Muscle Res Cell Motil 23: 535-540.

Wildenberg, G., M. Dohn, R. Carnahan, M. Davis, N. Lobdell, J. Settleman (2006) p120-catenin and p190RhoGAP regulate cell-cell adhesion by coordinating antagonism between Rac and Rho. Cell 127: 1027-1039.

Yilmaz, M., F. Christofori (2009) EMT, the cytoskeleton, and cancer cell invasion. Cancer Metast Rev 28: 15-33.

Zaman, M., R. Kamm, P. Matsudaira, D. Lauffenburger (2005) Computational model for cell migration in three-dimensional matrices. Biophys J 89: 1389-1397. 